\title{
ON THE PREVALENCE OF EVENT CLUSTERS IN AUTOBIOGRAPHICAL MEMORY
}

\author{
Norman R. Brown \\ University of Alberta, Edmonton, AB, Canada
}

\begin{abstract}
Event clusters are narrative-like memory structures that draw together information about causally and thematically related events (Brown \& Schopflocher, 1998a, 1998b). Prior research, using a method called event cueing, indicates that clusters play an important role in the organization of autobiographical memory and suggests that cluster formation is a by-product of the normal processes required to plan, execute, evaluate, and discuss meaningful event sequences. Two new event-cueing experiments are reported in this article. In the first, participants (undergraduates) were cued with personal memories from the past week, the recent past (high school), or the distant past (grade school) and were required to respond to each of the cueing events by retrieving a second, related personal event (the cued event). Cueing and cued events were often drawn from the same cluster. However, cueing events from the distant past were somewhat less likely to elicit clustermates than cueing events from the past week or the recent past. Experiment 2 demonstrated that participants responded to cueing events fastest when they were instructed to retrieve clustermates and slowest when they were required not to retrieve clustermates. These findings rule out biased retrieval as an explanation for the frequent recall of clustermates and suggests that the cluster rates provide a conservative measure of the prevalence of event clusters in autobiographical memory.
\end{abstract}

Event clusters are narrative-like memory structures that draw together information about events that are causally or thematically related (Brown \& Schopflocher, 1998a, 1998b). Prior research indicates that these clusters play an important role in the organization of auto-

This research was supported by the author's NSERC operating grant. I would like to thank Don Schopflocher for his many contributions to the event-cueing project and Courtney Bryden and Peter Lee for their comments on this article. Correspondence should be addressed to Norman R. Brown, Department of Psychology, University of Alberta, Edmonton, Alberta, T6G 2E9, Canada; E-mail: nrbrown@ualberta.ca. 
biographical memory and suggests that cluster formation is a by-product of normal processes required to plan, execute, evaluate, and discuss meaningful event sequences. Support for these claims has come from a series of experiments that have employed a method called event cueing (Brown \& Schopflocher, 1998a, 1998b; Fitzgerald, 1980; Sato, 2002; Wright \& Nunn, 2000). Two new event-cueing experiments are reported in this article. The aim of the first experiment was to assess the prevalence of event clusters across different life periods. The aim of the second was to determine whether cluster rates obtained using the event-cueing method provide an accurate index of the actual frequency of these structures in autobiographical memory. Before turning to these experiments, the event-cueing project is briefly reviewed.

The typical event-cueing experiment involves five tasks: an event-generation task, an event-cueing task, a relation-coding task, a dating task, and an importance-rating task. During the event-generation task, participants are required to recall a set of personal events and to provide a brief description of each. In some experiments, these events are elicited by word cues (Robinson, 1976; Rubin, 1982); in others, they are selected by the participants because they are considered to be particularly important. During the second task, the event descriptions generated during Task 1 are presented one at a time; and the participant is required to respond to each of these cueing events by recalling a second related event, the cued event. The third task requires participants to indicate how the cueing event and the cued event are related to one another. During this phase, each event pair is presented on the computer display along with a relation menu. This menu allows participants to indicate whether the cueing event and cued event took place in the same location, whether the two events involved the same people, whether they describe the same type of activity, whether one event caused the other, whether one event was part of the other, or whether both were part of some larger story. During the last two tasks, participants date and rate the importance of each of the cueing and cued events.

Responses collected during the relation-coding task, or Task 3, are used to determine the clustering status of each event pair. Events are considered to be members of the same cluster only when the participants indicate that the two events were causally related, that one pair member was nested within the other, or that both members were part of the same broader story. Clustered pairs differ from nonclustered 
pairs in several ways: Cued events are retrieved more rapidly when the cueing event accesses a clustermate than when it does not; the events in clustered pairs tend to be more similar in age than events in nonclustered pairs; and cued and cueing events are more likely to refer to the same people, locations, and activities when the events are members of the same cluster than when they are not. In addition, under most conditions, clustered pairs tend to be far more common than nonclustered pairs, though cueing events are more likely to elicit clustermates when they are important than when they are not.

These core findings have three implications. First, the prevalence of event clusters revealed by event-cueing experiments indicates that these story-like structures play a central role in organizing autobiographical memories. Second, the pervasiveness of these structures, regardless of the importance of the cueing event, suggests that cluster formation is a by-product of normal processes required to plan, execute, and evaluate meaningful action sequences (Hayes-Roth \& Hayes-Roth, 1979; Roese \& Olson, 1995; Trabasso \& van den Broek, 1985). Third, the relation between importance and clustering suggests that important events receive additional memory-relevant processing, processing not allotted to events of a more mundane type.

One obvious source of this additional processing is narration. Certainly, people create and recount stories that describe significant or interesting personal experiences (e.g., Bruner, 1991; Bruner \& Feldman, 1996; Robinson \& Taylor, 1998). In principle, the composition and communication of these personal narratives should have mnemonic consequences. The act of composing (or modifying) a personal narrative is likely to reinforce preexisting interevent associations when they are considered important and to prune them when they are not. The communication of an existing narrative can be seen as a form of rehearsal, one that impedes forgetting of the story's constituent events and the relations that hold between them. Given that important events are more likely to undergo these narrative processes than unimportant events (Anderson \& Conway, 1993; Burt, Mitchell, Raggatt, Jones, \& Cowan, 1995), it follows that the former should cue clustermates more often than the latter.

In brief, the pervasiveness of event clusters suggests that these structures are created as a matter of course when people plan, execute, and evaluate goal-directed action sequences. The relationship between cueing-event importance and clustermate retrieval sug- 
gests that clusters may be subjected to differing amounts of post-event narrative processing. These claims constitute the matter-of-course position. This position and the research that supports it are consistent with the widely held belief that narrative-like structures are "a primary form of organization in autobiographical memory" (Robinson, 1992, p. 223; also see Barsalou, 1988; Brown, 1990; Bruner, 1991; Conway, 1996; Fivush, 1991; Linton, 1986; Nelson, 1993; Schank \& Abelson, 1995). At the same time, this approach differs in important ways from other prominent perspectives on event memory. One of these assumes that socially mediated narrative interactions are essential for the creation and maintenance of autobiographical memories (Barclay, 1996; Fivush, 1991; Nelson, 1993; Schank \& Abelson, 1995). If so, one would expect that important and/or interesting events (i.e., events worth recounting or discussing) are frequently embedded in event clusters and that unimportant and/or uninteresting events are not. However, as noted above, event clusters are common even when their constituent memories are mundane and thus unlikely to attract comment (Brown \& Schopflocher, 1998b; Experiment 1 below). This suggests that the formation of these clusters does not depend on overt rehearsal and/or social mediation.

Another approach to autobiographical memory assumes that events are organized around the concepts they embody. In particular, Schank and colleagues have argued that "action concepts" play a central part in structuring autobiographical knowledge (Kolodner, 1983; Reiser, 1983; Reiser, Black, \& Abelson, 1985; Schank, 1982). This position predicts that memories in event pairs almost always would involve the same type of action and that same-action pairs should be much more common than pairs that overlap in other ways. Instead, event-cueing experiments consistently demonstrate that paired events share persons and locations at least as often as they involve the same type of activity. These experiments also demonstrate that no single relation dominates when people indicate what the event pairs have in common. Thus, it seems unlikely that autobiographical memory is organized by actions concepts or by any other single conceptual class (e.g., persons, locations, emotions, etc.).

Experiment 1 was designed to provide additional evidence for the matter-of-course position by examining cueing-event age and cluster prevalence-an issue not addressed directly by prior event-cueing experiments. In this experiment, participants were re- 
quired to generate cueing events from the past week, the recent past (high school), or the distant past (grade school). If clustering is a by-product of normal event processing, as the matter-of-course position contends, and if this type of event processing occurs in childhood, adolescence, and (young) adulthood, then clustering should be common regardless of the age of the cueing events.

This prediction and the interpretation of prior event-cueing experiments presuppose that the percentage of retrieved clustermates provides an accurate indication of the proportion of event memories that are embedded in event clusters. There are, however, other plausible explanations for the high rate of clustering obtained using the event-cueing method. One possibility is that people are biased to search for memories that are part of the same story as the current event memory (perhaps because people are highly practiced at telling stories or because they are using script-like structures to generate retrieval cues) and that they consider other types of memories only when the search for a same-story event fails. If people use this biased two-stage search process, they should retrieve more clustermates than non-clustermates, they should access the former more rapidly than the latter, and this pattern should hold regardless of how event memories are organized. Experiment 2 provides a test of this biased-search hypothesis.

\section{EXPERIMENT 1}

The current experiment was designed to assess the relationship between event age and clustering. To do this, data were collected from three groups using the standard five-task event-cueing method. What differentiated one group from the others were restrictions that defined the age of an acceptable response in Task 1, the word-cued event-generation task. Specifically, participants in the past-week group were required to respond to each cueing word with a specific personal memory that happened during the past week. Participants in a second group, the recent-past group, were required to recall events that were at least one year old and no more than five years old. Because the people who took part in this experiment were first- and second-year undergraduates, the recent-past target period overlapped with the years these students were in high school. Participants assigned to the third group, the distant-past group, were instructed to retrieve events that took place during childhood. In this condition, recall was restricted to events that happened no earlier 
than the participant's fourth birthday and no later than his or her thirteenth birthday.

As noted above, the matter-of-course position contends that clustering is largely a consequence of normal event processing. If so, cueing events, regardless of their age, should frequently facilitate access to clustermates. Underlying this prediction is the recognition that actions we take on a daily basis are often goal-directed and/or reactive and that children regularly engage in similar planful action sequences. Of course, there also are important differences between the time periods probed in this experiment and these differences might have organizational consequences. For example, in the past-week condition, some of the memories retrieved during the word-cueing task may refer to events that have not yet produced the reactions they eventually will. In these cases, the cueing event might fail to access a clustermate not because the normal event processes do not create event clusters for mundane events but because the events that will eventually be subsumed by an event cluster have not yet occurred. Clustering might also be less common in the past-week condition than in the recent-past condition because very recent cueing events are often too mundane to warrant discussion or reevaluation and thus are unlikely to elicit the mnemonically relevant post-event processing.

It is also possible that event cues from the distant past might access fewer clustermates than those from the more recent past. This might happen because participants have forgotten some of the events that were initially associated with the cueing event or because people process events somewhat differently in childhood than they do later in life. Thus, although the matter-of-course position predicts that event clusters should be common in each of the three conditions studied in this experiment, there were reasons to expect that the retrieval of clustermates might be affected by the age of the cueing event.

\section{METHOD}

\section{PROCEDURE}

Data were collected from three groups: a past-week group, a recent-past group and a distant-past group. Regardless of condition, participants performed the same 5 tasks, in the same order. During Task 1, the word-cueing task, all participants were presented with 14 
nouns (food, store, clothes, house, animal, friend, work, money, family, vehicle, furniture, game, music, restaurant) and asked to respond to each with the first personal event that came to mind, provided that it was related to the cueing word and that it happened during the life period specified in the instructions. At the beginning of this task, participants were told that the retrieved memory should refer to a specific personal event that lasted at least a few minutes but no more than a few hours. In the past-week condition, participants were required to respond with memories that referred to events that were at least 24 hrs old but no more than seven days old. In the recent-past condition, participants had to respond to each cueing word with an event memory that was at least a year old but no more than five years old. In the distant-past condition, the events had to be drawn from a period that was bounded by the participant's fourth and thirteenth birthdays.

Event descriptions generated during Task 1 served as retrieval cues during Task 2. During the second task, participants were required to respond by retrieving the memory of a personal event that was somehow related to the cueing event. The instructions noted that the cued and cueing event might be related in a number of ways and warned participants that they should not respond with evaluative statements, trivial details, descriptions of their emotional states during the event, or generic event descriptions.

With the exception of these differences, the procedures followed during Tasks 1 and 2 were identical. Participants initiated a trial by pressing the ENTER key on a computer keyboard in response to a message presented on a computer-controlled video display. When the ENTER key was pressed, the initiation prompt was replaced by a cueing word (Task 1) or an event cue (Task 2). Participants were instructed to press the SPACE BAR as soon as they had an appropriate event memory in mind, but not before. Pressing the SPACE BAR caused an 80-character wide response field to appear on the computer screen. Participants typed a description of the recalled event in this field and then pressed the ENTER key to complete the trial. This procedure made it possible to separate retrieval time (RT) from typing time. On each trial, RT was measured from the onset of the cue word or event cue until the participant pressed the SPACE BAR. If a participant failed to respond to the cue within $90 \mathrm{~s}$, the trial was terminated.

On each trial during Task 3, participants were presented with an event pair (i.e., a cueing event and the cued event it elicited) and the 
relation menu. The menu listed the following questions on separate lines: Did Event A (the cueing event) and Event B (the cued event) involve the same person or persons? Did Event $A$ and Event $B$ involve the same activity? Did Event A and Event B occur at the same location? Did one of the events cause the other? Is one of the events part of the other? Are both of these events part of a single broader event? Are Event A and Event B related in some other way? Participants responded to each question by typing $\mathrm{Y}(\mathrm{es})$ or $\mathrm{N}(\mathrm{o})$ in the appropriate response field.

During Tasks 4 and 5, participants were presented with the event descriptions generated during the first and second tasks. During Task 4, participants estimated the day, month, and year when each of the recalled events occurred. During Task 5, they rated the personal importance of each event on a 1 (not important at all) to 5 (extremely important) scale. Tasks 3, 4, and 5 were untimed and self-paced.

The first two events recalled during Task 1 served as practice items during Task 2, and the event pairs made from the practice items appeared first during Task 3. During Tasks 4 and 5, the cueing and cued events from the two practice pairs were randomized and presented on the first four trials. Otherwise, cueing events were presented in a random order during Task 2 and the event pairs were presented in a random order during Task 3. Cueing and cued events were presented randomly in Tasks 4 and 5, with the constraint that when one member of a pair appeared during the first half of the task, the other appeared during the second.

\section{PARTICIPANTS}

One hundred and seventy-four University of Alberta undergraduates took part in this experiment. Participants were assigned at random to one of the three groups, with the constraint that an equal number (58) were placed in each. All were Introductory Psychology students who received course credit for their cooperation, and each was tested individually in a session lasting from 1.5 to $2 \mathrm{hrs}$.

\section{RESULTS}

The results of this experiment are reported in three parts: the first deals with the effect of the age restriction on the cue-word task, the second provides an overview of between-group differences, and the 
third focuses on the relationship between cluster status and performance.

Because responses from the practice trials were eliminated, each participant could contribute data from a maximum of 12 event pairs. Prior to conducting any of the analyses reported below, a pair of coders read through all (non-practice) event descriptions generated during Task 1 and Task 2 and classified each as either "acceptable" or "unacceptable." Acceptable responses were ones that referred to specific autobiographical events, whereas unacceptable ones described recurring events, emotional states or reactions, minor details of the cueing event, or general aspects of the self or the world.

\section{PERFORMANCE ON THE WORD-CUEING TASK}

Overall, participants in the distant-past condition had more difficulty recalling specific event memories in response to the word cues than did those in the recent-past condition or the past-week condition. This difficulty manifested itself in two ways. First, it took longer to retrieve event memories in the distant-past condition than in the other two conditions; second, when memories from the distant past were retrieved, they were less likely to refer to specific events than were memories from the more recent periods.

These claims are based on two one-way ANOVAs, one performed on median RTs and the other on the unacceptable-response percentages. In both cases, each participant in each group contributed a median RT, which was computed over all acceptable and unacceptable responses, ${ }^{1}$ and an unacceptable-response percentage, which was computed by dividing the number of unacceptable responses produced by a given participant by 12 .

On average, median RT in Task 1 increased from $5.71 \mathrm{~s}$ in the past-week condition to $7.64 \mathrm{~s}$ in the recent-past condition, to $9.00 \mathrm{~s}$ in the distant-past condition, $F(2,171)=5.21, p<.01, M S E=30.38$. Of the past-week responses, $11 \%$ were considered unacceptable, as were $7 \%$ of the recent-past responses, and $16 \%$ of the distant-past responses, $F(2,171)=4.76, p=.01, M S E=2.20$. A set of Least-Squares

1. Time-outs were uncommon in this task, occurring on $2 \%$ of the past-week trials, $1 \%$ of the recent-past trials, and $3 \%$ of the distant-past trials, $F<1.0$. 
Differences $(L S D)$ tests conducted on the RT data indicated that the distant-past medians were significantly slower than the medians observed in the past-week and recent-past conditions and that the retrieval times in the recent-past condition were not significantly slower than those in the past-week condition. ${ }^{2}$ LSD tests performed on unacceptable-response percentages indicated that participants in the distant-past group also were reliably less likely to respond to word cues with acceptable event memories than were those in the recent-past group. By this measure, performance in the past-week condition was not significantly better than performance in the distant-past condition nor was it significantly worse than it was in the recent-past condition.

\section{OVERVIEW OF PERFORMANCE ON THE EVENT-CUEING TASK}

Participants sometimes failed to recall an event memory of any sort in response to a word cue or cueing event. They also sometimes provided unacceptable responses in Tasks 1 and 2. As a result, it was necessary to groom the data set before analyzing the event-cueing task and characterizing the event pairs. This was done in three steps. First, data were not considered when a participant timed out during Task 1 and responses concerning cueing events were discarded when the participant timed out during Task 2. Second, both events in a pair were discarded when either was judged to be unacceptable. Third, all data obtained from participants who produced fewer than six (of 12) acceptable event pairs were excluded. The process resulted in the elimination of 12 participants from the past-week group, three from the recent-past group, and 12 from the distant-past group. On average, each of the remaining 46 past-week participants produced 8.37 acceptable event pairs, each of the remaining 55 recent-past participants produced 9.78 acceptable event pairs, and each of the remaining 46 distant-past participants produced 9.09 acceptable event pairs, $F(2,144)=8.12, p<.001, M S E=3.08$. In other words, 385, 538, and 411 acceptable pairs were collected from retained participants

2. The same pattern holds when medians were computed for acceptable responses alone. In this case the average median RTs are $5.67 \mathrm{~s}, 7.67 \mathrm{~s}$, and $9.24 \mathrm{~s}$ for the past-week, recent-past, and distant-past groups, respectively, $F(2,171)=5.68, p<.01, M S E=32.84$. 
in past-week, recent-past, and distant-past conditions, respectively. ${ }^{3}$

Data associated with acceptable event pairs were used to compute the following measures for each retained participant in each of the three groups: median Task 1 RT, median Task 2 RT, mean rated importance for cueing events, mean rated importance for cued events, median cueing event age, median cued event age, and a median age difference (event age difference $=\mid$ cueing-event age - cued-event age I). A cluster rate also was computed for each of the retained participants. A positive response to the causal question, the nested question, or the same-story question during the relation-coding task (Task 3) was taken as an indication that the events in the pair were drawn from the same event cluster. This criterion was used to determine, for each participant, the percentage of acceptable event pairs composed of clustermates. Separate one-way (group: past-week vs. recent-past vs. distant-past) ANOVAs (and LSD tests when appropriate) were performed on each measure.

Table 1 presents group averages for these measures and indicates which ANOVAs yielded reliable between-group differences. There are two important things to notice about these data. First, in this experiment, as in prior ones (Brown \& Schopflocher, 1998a, 1998b), clustering was common, with clustermates being retrieved more than half the time regardless of the age of the cueing event. Second, clustering was not equally common across conditions, $F(2,144)=$ $3.68, p<.05, M S E=6.18$. In particular, event cues from the distant past $(54 \%)$ were reliably less likely to elicit clustermates than those from the recent past $(69 \%)$. The average cluster rate for the past-week group (63\%) fell between the two other rates, but was not reliably different from either. ${ }^{4}$

3. Given that event memories from the very recent past are highly accessible (Brown \& Schopflocher, 1998a; Nelson, 1993; Reiser, 1983), it is interesting that participants in the past-week condition had more difficulty producing acceptable event pairs than did participants in the recent-past and distant-past conditions. One possible explanation for this is that the experiences confined to any given week are necessarily more limited than those that occurred over a five or ten year span. As a result, it is less likely that a given cueing word would be related to an event that happened in the past week than one that happened during the periods defined in the other two conditions.

4. These mean cluster rates were very similar to the raw cluster rates, which were computed for each condition by dividing the total number of clustered pairs by the total number of acceptable pairs. The raw cluster rates were $63 \%, 69 \%$, and $54 \%$ for the past-week, recent-past, and distant-past conditions respectively, $\chi^{2}(2, N=1334)=17.24, p<.001$. 
TABLE 1. Experiment 1 Averages and Standard Errors (in parenthesis), Computed Over Participants, for MD Task 1 and Task 2 RTs, MD Task 1 and Task 2 Event Age, MD Age Difference, Mean Task 1 and Task 2 Rated Importance, and Percent Clustered

\begin{tabular}{|c|c|c|c|}
\hline & \multicolumn{3}{|c|}{ Cueing Event Periods } \\
\hline & Past Week & Recent Past & Distant Past \\
\hline Task 1 RT (s)* & $6.11(0.60)$ & $7.38(0.71)$ & $8.93(0.74)$ \\
\hline Task 2 RT (s) ${ }^{\text {ns }}$ & $8.93(0.88)$ & $9.64(0.91)$ & $10.87(1.09)$ \\
\hline Task 1 Event Age (days ago) ${ }^{* * * *}$ & $3.36(0.19)$ & $637.81(43.68)$ & $4141.52(142.20)$ \\
\hline Task 2 Event Age (days ago) ${ }^{* * * *}$ & $104.93(36.43)$ & $537.83(43.21)$ & $3064.91(235.93)$ \\
\hline Age Difference (days) $)^{* * * *}$ & $101.95(36.46)$ & $174.95(29.77)$ & $957.34(173.65)$ \\
\hline Task 1 Rated Importance ${ }^{* *}$ & $2.59(0.10)$ & $2.89(0.08)$ & $2.44(0.10)$ \\
\hline Task 2 Rated Importance ${ }^{\text {ns }}$ & $2.78(0.09)$ & $2.63(0.08)$ & $2.68(0.09)$ \\
\hline$\%$ Clustered* & $62.91(3.65)$ & $67.15(2.98)$ & $53.83(4.12)$ \\
\hline
\end{tabular}

Note. Superscript symbols denote significance levels derived from a one-way (between-group) ANOVA (with 2 and 144 degrees of freedom) performed on the indicated measure. Significance levels associated with these symbols are as follows: $\mathrm{ns}=p>.10 ;+=0.10<p<0.05 ;{ }^{*}=p<.05 ;{ }^{* *}=p<.01 ;{ }^{* * *}=p$ $<0.001 ; * * * *=p<0.0001$

In addition, it is worth noting that the age of the cueing events increased from 3.36 days in the past-week condition to 637.81 days in the recent-past condition to 4141.52 days in the distant-past condition, $F(2,144)=704.02, p<.0001, M S E=330036.81$. This provides a simple manipulation check, as it indicates that event retrieval was directly affected by the constraints included in the Task 1 instructions. The average median age of the cued events and the average median difference between cueing and cued events also increased across conditions: for cued-event age, $F(2,144)=139.47, p<.0001, M S E=857733.12$, and for event age differences, $F(2,144)=22.49, p<.0001, M S E=470837.55$.

Also of some interest are the importance ratings, which suggest that participants in all groups considered the events they retrieved in Task 1 and Task 2 to be moderately important, with recent-past cueing events receiving higher ratings than cueing events from the past week and the distant past: for cueing events, $F(2,144)=6.26, p<.01, M S E=$ 0.41 , and for cued events, $F<1.0$. Finally, the analysis of median Task 1 RTs, which in this case was restricted to trials associated with acceptable event pairs produced by retained participants, parallels the more inclusive ANOVA described above: as before RT increased with event age, $F(2,144)=3.89, p<.05, M S E=23.56$. Although Task 2 RTs also in- 
creased across conditions, this difference was not significant, $F<1.0$. Event age and RT are considered further below.

\section{CLUSTER-STATUS EFFECTS}

The two primary goals of the present study were to replicate previously reported cluster-status effects (Brown \& Schopflocher, 1998a, $1998 \mathrm{~b}$ ) and to determine whether event age influences these effects. Data bearing on these issues are presented in Table 2 and Figure 1. The RTs, event-age data, and importance ratings reported in these exhibits are medians computed separately over all acceptable clustered pairs and all acceptable nonclustered pairs produced by all retained participants from each of the three groups. Similarly, the relation-code percentages listed in Table 2 were computed separately over all acceptable clustered pairs and all acceptable nonclustered pairs. ${ }^{5}$

Two sets of statistical tests were conducted. One indicated, for each of the measures listed in Table 2, whether clustered pairs produced by participants in a given group differed reliably from the corresponding nonclustered pairs. For example, one of these tests indicated that participants in the past-week condition were more likely to specify that a cueing event and cued event took place in the same location when the events were drawn from the same cluster $(49 \%)$ than when they were not (31\%). The second set of statistics examined between-group differences separately for clustered pairs and nonclustered pairs. Returning to the location example, one of these tests indicated that the probability of providing a same-location response to clustered pairs was unaffected by the age of the cueing event. A second test indicated that the same was true for same-location responses elicited by nonclustered pairs.

Kruskal-Wallis tests were used to determine whether observed differences in RT, event dating, and rated importance were reliable. Simple $\chi^{2}$ tests performed the same function for the data collected during the relation-coding task. Significance levels obtained from these tests are indicated in Table 2 by the superscript letters suffixed to the cluster-pair values and by the superscript symbols suffixed

5. In what follows, participant was not used as the unit of analysis because some individuals only produced clustered pairs, whereas others produced very few. 


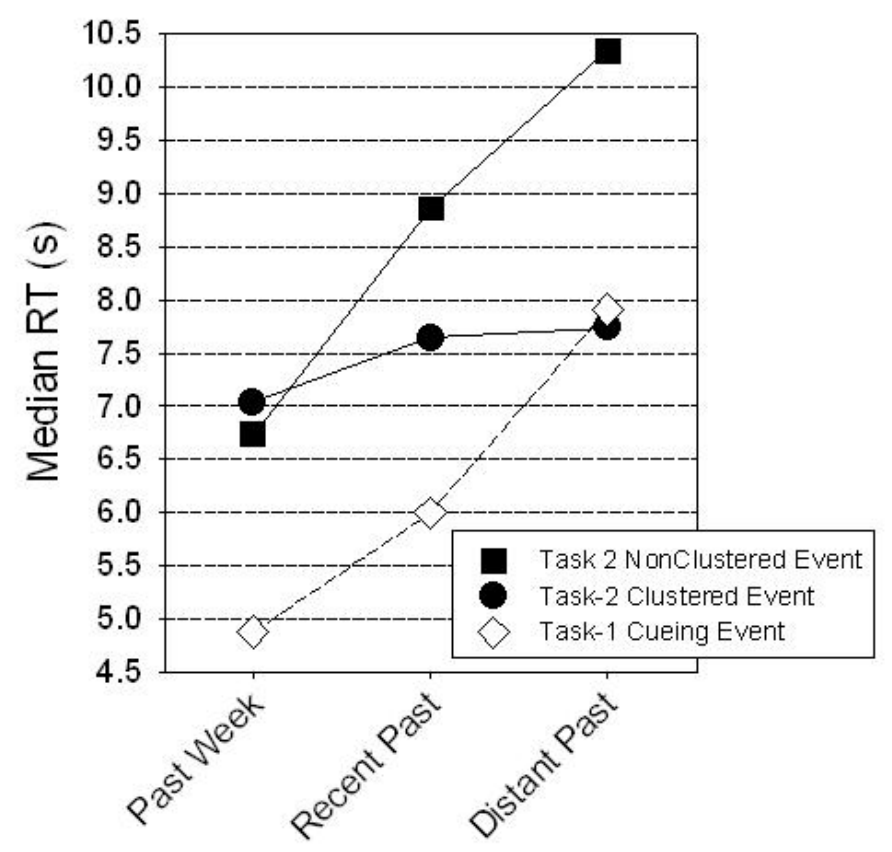

FIGURE 1. Median Response Times (RTs) as a function of the event age, for the retrieval of acceptable cueing events in Task 1 (unfilled diamonds) and acceptable clustered events (filled circles) and nonclustered events in Task 2 (filled square). Experiment 1 data.

to the row stubs. The superscript letters indicate reliable within-group cluster-status effects and the superscript symbols indicate reliable between-group effects obtained holding cluster-status constant.

The results presented in Table 2 can be summarized quite simply. With a few minor exceptions, the current experiment replicates the clustering effects observed in prior studies and indicates that event clusters from the past week, the recent past, and the distant past are much the same. As noted above, prior event-cueing experiments have demonstrated that: (a) clustered events tend to occur close together in time and nonclustered events do not; (b) clustered pairs are more likely than nonclustered pairs to refer to the same people, take place at the same location, and involve the 
same activity; (c) participants retrieve cued events faster when the cueing and cued event are part of the same cluster than when they are not; and (d) important cueing events are more likely to elicit clustermates than less important cueing events (Brown \& Schopflocher, 1998b). Data relevant to each of these cluster-status effects are presented below.

Cluster Status and Dating Differences. It is obvious from data presented in Table 2 that between-event temporal differences increased sharply with the age of the cueing events, and that this was true regardless of cluster status: for clustered pairs, Kruskal-Wallis test $\chi^{2}(2, N=822)=82.29, p<.0001$, and for nonclustered pairs, Kruskal-Wallis test $\chi^{2}(2, N=512)=135.58, p<.0001$. More importantly, temporal proximity and cluster status were related in each condition. Specifically, clustered events differed by 0 days, 6.5 days, and 92.0 days in the past-week, recent-past, and distant-past conditions, respectively. Comparable differences for nonclustered events were 104.0 days, 361.5 days, and 2392.0 days: for the past-week condition, Kruskal-Wallis test $\chi^{2}(1, N=385)=135.58, p<.0001$, for the recent-past condition, Kruskal-Wallis test $\chi^{2}(1, N=538)=88.37, p<$ .0001 , and for the distant-past condition, Kruskal-Wallis test $\chi^{2}(1, N$ $=411)=115.64, p<.0001$.

Cluster Status and Coded Relations. To the extent that the date estimates are accurate, the between-group difference in the dates assigned to clustered events suggests that clusters from the three periods might be quite different. However, data from the relation-coding task indicated that event clusters from the three periods had much in common, whereas clustered pairs and nonclustered pairs seemed to be very different even when they were drawn from the same period. Consistent with prior research: (a) events in clustered pairs were more likely than those in nonclustered pairs to share the same people (for the past week, $50 \%$ vs. $34 \%, \chi^{2}(1, N=385)=9.10, p<.01$; and for the recent past, $53 \%$ vs. $38 \%, \chi^{2}(1, N=538)=11.75, p<.001$; for the distant past, $61 \%$ vs. $35 \%$, $\left.\chi^{2}(1, N=411)=28.37, p<.0001\right)$; (b) events in clustered pairs were more likely than those in nonclustered pairs to take place in the same location (for the past week, $49 \%$ vs. $31 \%, \chi^{2}(1, N=385)=12.53$, $p<.001$; for the recent past, $52 \%$ vs. $32 \%, \chi^{2}(1, N=538)=19.60, p<$ .0001 ; and for the distant past, $58 \%$ vs. $25 \%, \chi^{2}(1, N=411)=46.01, p<$ $.0001)$; and (c) events in clustered pairs were less likely than those in nonclustered pairs to be related by some relation not listed on the 


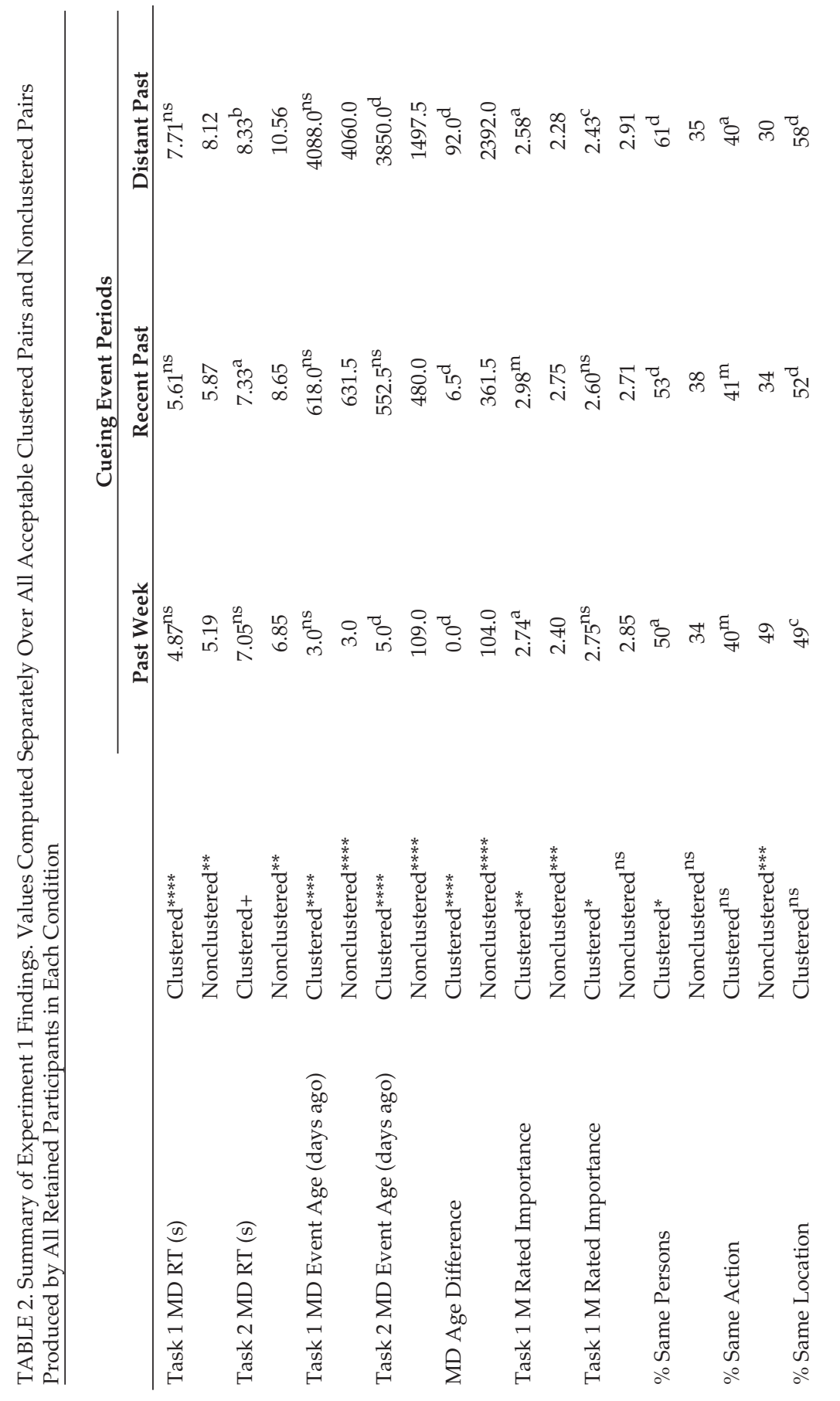




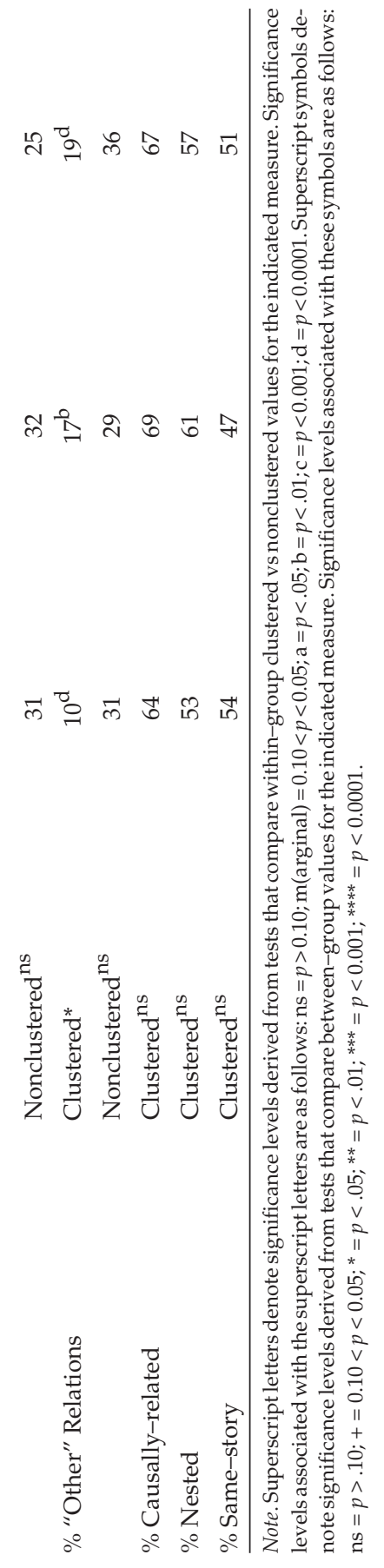


relation menu (for the past week, $10 \%$ vs. $31 \%, \chi^{2}(1, N=385)=26.30$, $p<.0001$; for the recent past, $17 \%$ vs. $29 \%, \chi^{2}(1, N=538)=9.38, p<$ .01 ; and for the distant past, $19 \%$ vs. $36 \%, \chi^{2}(1, N=411)=14.65, p<$ .0001). Also in keeping with prior research, clustered pairs were more likely than nonclustered pairs to involve the same type of activity for the recent-past condition $\left(41 \%\right.$ vs. $34 \%, \chi^{2}(1, N=538)=$ $2.96, .10<p<.01)$ and the distant-past condition $\left(40 \%\right.$ vs. $30 \%, \chi^{2}(1$, $N=411)=4.71, p<.05)$, but not in the past-week condition ( $40 \%$ vs. $\left.49 \%, \chi^{2}(1, N=385)=3.01, .10<p<.05\right)$.

The reverse cluster-status effect obtained for the activity relation in the past-week condition occurred because events in the nonclustered pairs (49\%) were more likely to involve the same activity than were those in the nonclustered pairs from the recent-past $(34 \%)$ and distant-past conditions $\left(30 \% ; \chi^{2}(2, N=512)=14.20, p<\right.$ .001). In contrast, the probability of a same-activity response for clustered pairs was essentially the same across the three time periods (for past week, $40 \%$, for recent past, $41 \%$; and for the distant past, $40 \%$; $\left.\chi^{2}(2, N=822)<1.0\right)$. Similarly, event age was unrelated to the probability that events in the clustered pairs would share a location (for past week, $49 \%$; for recent past, $52 \%$; and for the distant past, $58 \%$; $\left.\chi^{2}(2, N=822)=3.80, p>.10\right)$ or that they would be coded as being causally related (for past week, $64 \%$; for recent past, $69 \%$; for distant past, $\left.67 \% ; \chi^{2}(2, N=822)=1.73, p>.10\right)$, nested one within the other (for past week, $53 \%$; for recent past $61 \%$; for distant past, $57 \%$; $\chi^{2}(2, N$ $=822)=4.48, p=.10$ ), or part of the same broader stories (for past week $54 \%$; for recent past $47 \%$; for distant past $51 \% ; \chi^{2}(2, N=822)=$ $2.88, p>.10$ ). It is true that same-person responses (for past week, $50 \%$; for recent past, $53 \%$; for distant past, $61 \% ; \chi^{2}(2, N=822)=6.21, p$ $<.05$ ) and other-relation responses (for past week 10\%; for recent past $17 \%$; for distant past $\left.19 \% ; \chi^{2}(2, N=822)=8.99, p<.05\right)$ were most common when the clustered pairs occurred in the distant past. However, when compared to the many large cluster-status differences reported above, these between-group differences are quite modest.

Cluster Status and Retrieval Times. Taken as a whole, data from the relation coding task support the conclusion that event clusters from very different time periods are structured in much the same way. RTs from the event-cuing task (Task 2) provide additional support for this claim. Median retrieval times for the recovery of same-cluster and different-cluster events during Task 2 are plotted in Figure 1 (also see Table 2). For purposes of comparison, the median Task 1 
RTs, computed over all acceptable word-cued responses provided by all retained participants, are presented as well.

It is clear from this figure that the speed with which participants responded to the cueing events depended in an interactive manner on the age of the cueing event and the nature of the relation that held between the cueing event and the cued event. Replicating previous findings, clustermates were retrieved more readily than nonclustered event memories, at least when the cueing event came from the recent past (for clustered pairs, median RT $=7.33 \mathrm{~s}$; for nonclustered pairs, median RT $=8.85 \mathrm{~s}$; Kruskal-Wallis test $\left.\chi^{2}(1, N=538)=5.38, p<.05\right)$ or the more distant past (for clustered pairs, median RT $=8.33 \mathrm{~s}$; for nonclustered pairs, median RT $=10.56 \mathrm{~s}$; Kruskal-Wallis test $\chi^{2}(1, N=$ $411)=7.77, p<.01)$. Cluster-status was not reliably related to the retrieval times in the past-week condition (for clustered pair, median RT $=7.05 \mathrm{~s}$; for nonclustered pairs, median $\mathrm{RT}=6.85 \mathrm{~s}$; Kruskal-Wallis test $\left.\chi^{2}(1, N=385)<1.0\right)$. This pattern of differences emerged because Task 2 RTs were relatively fast and little affected by event age when the cueing and cued events were drawn from the same cluster, whereas RTs increased quite sharply across the conditions when the cueing event failed to access a clustermate; for clustered pairs, Kruskal-Wallis test $\chi^{2}(2, N=822)=4.76, p=.09$; for nonclustered pairs, Kruskal-Wallis test $\chi^{2}(2, N=512)=11.89, p<.01$.

The relatively flat RT function for clustermates is consistent with the idea that cueing events that elicit clustermates do so because they tap into preexisting memory structures and that retrieval from an accessed cluster is little affected by its age. In contrast, the increase in retrieval time observed when clustermates are not retrieved indicates that participants had more difficulty finding related event memories when the cueing events were old than when they were recent. As is apparent in Figure 1, this pattern closely parallels the one produced when participants in the three groups were responding to word cues during Task 1 , though the nonclustered Task 2 RTs were about 2.5 s slower than the Task 1 RTs.

The difference between the Task 1 RTs and the Task 2 nonclustered RTs is of little importance as it is probably related to the amount of reading required before the retrieval process can begin (i.e., one word in Task 1 vs. one sentence in Task 2). Of more interest is the similarity between the two RT functions because it suggests that the age-related factors that make the word-cueing tasks difficult are also at play in the event-cueing task. In the word-cueing task, event memories are occa- 
sionally retrieved directly from memory. More often, retrieval is mediated by an iterative process of cue-generation, fragment retrieval, and evaluation (Brown, 1993; Brown \& Schopflocher, 1998a; Norman \& Bobrow, 1979; Williams \& Hollan, 1981). It follows that the age-related increase in Task 1 RTs and (by analogy) in nonclustered Task 2 RTs may be due to a decrease in direct access to event memories across conditions, an increase in the number of retrieval cycles required to recall a related event memory, or both. Regardless, the RT differences indicate that recent memories are more accessible than older ones (Brown \& Schopflocher, 1998a; Nelson, 1993; Reiser, 1983), at least when the cue is not embedded in an event cluster.

Cluster Status and Rated Importance. The final issue addressed in this section concerns the relation between event importance and clustering. As in prior research, more important cueing events were more likely to elicit clustermates than less important ones. Collapsing over the three groups, clustering percentages for the five levels of rated importance were $54 \%, 60 \%, 64 \%, 62 \%$, and $77 \%, \chi^{2}(4, N=1334)$ $=24.04, p<.0001$. Although this general relationship between importance and clustering was apparent in each condition, a reliable effect of importance on clustering was obtained only in the distant-past condition. In this condition, clustering percentages for the five levels of rated importance were $46 \%, 56 \%, 58 \%, 45 \%$, and $78 \%, \chi^{2}(4, N=$ $411)=14.85, p<.01$. In the past-week condition, the corresponding percentages were $55 \%, 60 \%, 64 \%, 71 \%$, and $72 \%, \chi^{2}(4, N=385)=6.33$, $p>.10$. In the recent-past condition, they were $63 \%, 63 \%, 68 \%, 65 \%$, and $78 \%, \chi^{2}(4, N=538)=5.97, p>.1$. These results might suggest that the relationship between importance and clustering is not particularly robust. However, it is true that participants in each group were far less likely to retrieve a clustermate when the cueing events were considered to be extremely unimportant (i.e., when they received an importance rating of 1) than when they were considered to be extremely important (i.e., when they received an importance rating of 5). ${ }^{6}$ This suggests that some of the weakness in the relationship be-

6. Restricting the analyses to only those pairs that included extremely unimportant cueing events (rated importance $=1$ ) and extremely important cuing events (rated importance = 5), $\chi^{2}(1, N=139)=3.29, .05<p<.10$, for the past-week condition; $\chi^{2}(1, N=176)=$ $4.61, p<.05$, for the recent-past condition, and $\chi^{2}(1, N=173)=12.00, p<.001$, for the distant-past condition. 
tween importance and clustering may be due to the uncertainty associated with assigning importance ratings to event memories.

\section{DISCUSSION}

The main findings of Experiment 1 can be summarized as follows: (a) regardless of event age or importance, event memories were often embedded in event clusters; (b) like events in a story, clustered events, were often causally related, temporally proximate, and similar in content; (c) cueing events from the distant past were less likely to elicit clustermates than were cueing events from the past week or the recent past; (d) retrieval times were little affected by event age when the cueing event elicited a clustermate; (e) RTs increased with event age during the word-cueing task; they also increased in the event-cueing task when the cueing event failed to elicit a clustermate; and (f) Regardless of event age, important cueing events tended to elicit clustermates more often than unimportant cueing events.

For reasons developed above, the prevalence of event clusters across the three time frames, the similarities in their make-up, and the relationship between rated importance and clustering support the notion that event clusters are created as a matter of course as people plan for, engage in, reflect upon, and discuss goal-directed action. Unexpectedly, this experiment also demonstrated that event memories from the distant past are less likely to be embedded in event clusters than are more recent event memories. Because time from the present and the participants' age when the events occurred were confounded in this experiment, there are two ways to interpret this finding. One possibility is that children are somewhat less likely to create event clusters than are (young) adults. A second possibility is that forgetting from event clusters is graded and that, with the passage of time (and in the absence of rehearsal), event memories that were once embedded in event clusters come to "stand alone" in autobiographical memory. An additional experiment, one that manipulates the age of the cueing events and the age of the participants, will be necessary to select between these alternatives.

\section{EXPERIMENT 2}

Event-cueing studies indicate that it is common for retrievable event memories to be embedded in event clusters. This conclusion pre- 
sumes that the frequency of same-cluster responses observed in these event-cueing experiments provides an accurate reflection of the frequency of event clusters in long-term memory. Underpinning this accurate reflection assumption are several others. Specifically, it is assumed that interevent associations exist, that accessing one event memory facilitates retrieval of associatively related memories, and that people respond to event cues with the first event memory that comes to mind. It also is assumed that retrieval times are particularly fast when strong preexisting associations connect a cueing event to a cued event and that people must fall back on a laborious and time-consuming retrieval process (involving an iterative cycle of cue generation, fragment retrieval, and evaluation) when the cueing event is not directly associated with other event memories.

Although these assumptions seem reasonable, they have not been directly tested. This leaves open the possibility that the accurate-reflection assumption is itself incorrect and that event-cueing results do not provide a realistic index of cluster frequency. For example, it is possible that participants deliberately adopt a retrieval strategy that fosters the generation of clustermates. This could happen if people use event cues to access script-like knowledge structures (Shank \& Abelson, 1977) and then use script knowledge to search for (or construct) events of the sort that would typically precede or follow the current cueing event. Given that scripts organize information about common goal-directed action sequences, this strategy often would produce event pairs that would be classified as clustermates, regardless of whether the items in the pair are directly associated with one another.

If people adopt a script-based retrieval strategy or if they simply prefer to search first for events that make for a narrative type sequence, then obtained cluster frequencies might overestimate the frequency of these structures in autobiographical memory. Alternatively, it could be that the actual frequency of event clusters is underestimated by the event-cueing method. Underestimation is a potential problem because participants are required to respond to each cueing event with a specific personal event that is related in some way to the cueing event, and they are prohibited from responding with minor details, emotional reactions, and the like. Given these requirements, it is clear that each retrieved memory has to be evaluated on a number of criteria before it could be put forward as a response. This raises the possibility that some legiti- 
mate event memories (directly) accessed by the cueing event might be rejected during the evaluation stage. If so, cluster frequencies obtained from event-cueing experiments might be best understood as providing a conservative measure of the actual prevalence of event clusters.

The current experiment, which used a variant of the standard five-task event-cueing method, was designed to determine whether empirically derived cluster frequencies provide an accurate index of underlying cluster prevalence. In this experiment, data were collected from three groups: a same-narrative group, a non-narrative group, and a neutral group. What distinguished the groups from one another is the instructions participants received prior to the event-cueing task, Task 2. During the event-cueing task, participants in the same-narrative group were required to recall an event that came from the "same story" as the cueing event. In contrast, participants in the non-narrative group were instructed to report the first event memory that came to mind, provided that it was related to the cueing event but "not part of the same story." Finally, in the neutral condition, participants were instructed to respond to the cueing event with the first related event memory that came to mind. In this condition, no restrictions were placed on the type of relationship that should or should not be obtained between the cued and cueing events.

The experiment was undertaken with the expectation that the usual pattern of cluster-status differences would be observed in the neutral condition. This would mean that Task 2 RTs should be faster when participants retrieve same-cluster events than when they do not, that events in clustered pairs should be very close in age, and that events in clustered pairs should share event components more often than events in nonclustered pairs.

Beyond providing an opportunity to replicate these cluster-status differences, the current design made it possible to observe the effect of retrieval instructions on Task 2 RTs. If the cluster frequencies accurately reflect the relations that hold between event memories and if the processing assumptions that support this view are correct, then:

(a) Task 2 RTs produced when non-narrative participants retrieve nonclustered events should be slower than the comparable RTs collected from participants in the neutral group, and (b) Task 2 RTs produced when same-narrative participants retrieve clustered events 
should be the same as comparable RTs collected from participants in the neutral group.

The first prediction assumes that event memories frequently are embedded in event clusters and that processing one of these cluster-embedded events during the event-cueing task often provides direct access to other clustermates. If these assumptions are correct, participants in the non-narrative condition will sometimes encounter clustermates during Task 2 . These memories will then have to be evaluated and discarded before a deliberate memory search is initiated. In other words, direct access to information in the cueing event's event cluster should interfere with the ability of participants in the non-narrative condition to retrieve nonclustered event memories. Alternatively, it could be that people are biased to search for (or construct) same-narrative events and that they seek other types of event memories only when this first process fails. This biased-retrieval position predicts that the retrieval of nonclustered event memories during Task 2 should be faster in the non-narrative condition than in the neutral condition. In this case, the nonclustered RTs in the non-narrative condition should be faster than those in the neutral condition because participants in the non-narrative condition immediately should seek nonclustered event memories, whereas those in the neutral condition should begin this secondary search of memory only after they have unsuccessfully completed a time-consuming search for a clustermate.

The second prediction, that the retrieval of same-cluster memories will be no faster in the same-narrative condition than in the neutral condition, assumes that participants respond with the first event memory that comes to mind. However, for reasons outlined above, participants in the neutral condition might screen information with some care. This screening process, combined with the occasional rejection of a recovered event memory (which necessitates additional search and evaluation), would increase the average amount of time required to respond to an event cue. It could be that retrieved information, particularly information that is closely related to the cueing event, is screened less carefully by same-narrative participants than by neutral-instruction participants. If so, same-cluster Task 2 RTs should differ between groups, with the participants in the same-narrative group responding more rapidly than those in the neutral group. 


\title{
METHOD
}

\author{
PROCEDURE
}

With two exceptions, the procedure used in this experiment was identical to the one used in Experiment 1. One difference concerned Task 1 instructions. In contrast to Experiment 1, no temporal restrictions were placed on the age of the event memories retrieved in response to the word cues. In this experiment, participants were free to respond to the word cues with memories from any period of their lives. The second difference concerned requirements imposed during Task 2. In this experiment, participants in the same-narrative group were instructed to respond only if they could remember an event that was part of the same story as the cueing event, and participants in the non-narrative group were instructed to respond only if they could remember an event that was related to the cueing event but was not drawn from the same story. As in Experiment 1, the instruction in the neutral condition required that cueing and cued events be related, but did not specify how they should be related.

\section{PARTICIPANTS}

One-hundred and ninety-six University of Alberta Introductory Psychology students took part in this experiment. Participants, who received course credit for their cooperation, were randomly assigned to conditions (with 66 per group) and were tested individually in sessions lasting from 1.5 to $2.0 \mathrm{hrs}$.

\section{RESULTS}

As in Experiment 1, data were lost when the participants failed to respond to the cueing word or the cueing event within 90 s. In addition, all event descriptions were classified as being acceptable or unacceptable and event pairs were eliminated when one (or both) of the descriptions was unacceptable. Finally, all responses from participants who produced fewer than six acceptable event pairs were excluded from further analysis. These procedures resulted in the elimination of five participants in the neutral condition, five in the same-narrative condition, and three in the non-narrative condition. On average, retained participants generated 10.1 acceptable event pairs in the neutral condition and 10.5 in both the same-narrative 


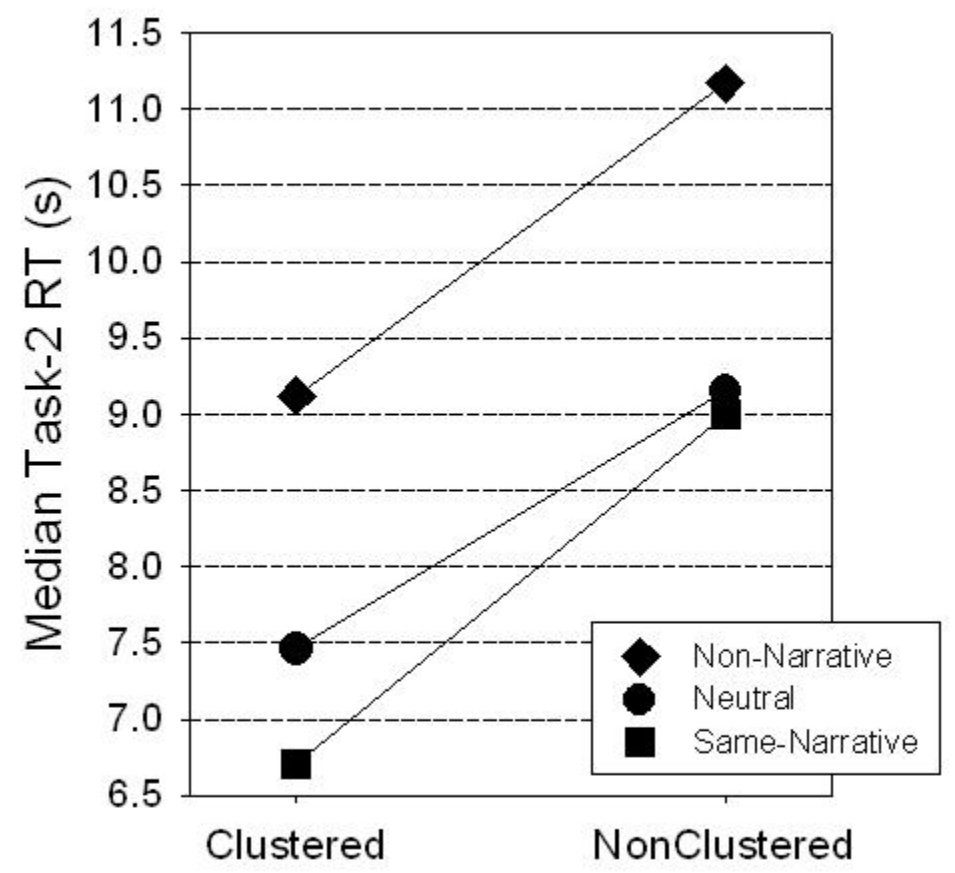

FIGURE 2. Median Responses Times (RTs) as a function of cluster status, for the retrieval of acceptable cued events during Task 2 in the same-narrative condition (squares), the neutral condition (circles), and the non-narrative condition (diamonds). Experiment 2 data.

and non-narrative groups $(F(2,182)=1.37, p>.10, M S E=2.69)$, yielding a total of 613,641 , and 662 acceptable event pairs across the three conditions.

Data associated with acceptable event pairs generated by the retained participants were subjected to the same treatment in Experiment 2 as in Experiment 1. The information presented in Table 3 provides an overview of performance that takes participants as the unit of analysis. Table 4 presents the medians, means, and percentages computed separately over clustered pairs in the neutral and same-narrative conditions and over the nonclustered pairs in the neutral and non-narrative conditions. (Values from the clustered pairs obtained in the non-narrative condition and nonclustered 
TABLE 3. Experiment 2 Averages and Standard Errors (in parenthesis), Computed Over Participants, for MD Task 1 and Task 2 RTs, MD Task 1 and Task 2 Event Age, MD Age Difference, Mean Task 1 and Task 2 Rated Importance, and Percent Clustered

\begin{tabular}{|c|c|c|c|}
\hline & \multicolumn{3}{|c|}{ Task 2 Instructions } \\
\hline & Neutral & Same-Narrative & Non-Narrative \\
\hline Task 1 RT (s) ${ }^{\text {ns }}$ & $7.80(0.66)$ & $7.53(0.58)$ & $6.88(0.49)$ \\
\hline Task 2 RT $(\mathrm{s})^{* *}$ & $9.40(0.71)$ & $8.74(0.81)$ & $12.75(0.93)$ \\
\hline Task 1 Event Age (days ago) ${ }^{\text {ns }}$ & $1331.34(152.23)$ & $1298.97(192.22)$ & $1006.59(128.23)$ \\
\hline Task 2 Event Age (days ago)* & $1244.25(146.24)$ & $1284.93(193.62)$ & $798.13(113.49)$ \\
\hline Age Difference (days) ${ }^{* * * *}$ & $212.94(47.05)$ & $100.50(88.47)$ & $606.16(91.63)$ \\
\hline Task 1 Rated Importance* & $3.14(0.07)$ & $3.29(0.08)$ & $3.00(0.07)$ \\
\hline Task 2 M Rated Importance ${ }^{\text {ns }}$ & $3.07(0.07)$ & $3.02(0.08)$ & $3.05(0.07)$ \\
\hline$\%$ Clustered ${ }^{* * * *}$ & $67.74(3.46)$ & $93.49(1.60)$ & $31.02(2.80)$ \\
\hline
\end{tabular}

Note. Superscript symbols denote significance levels derived from a one-way (between-group) ANOVA (with 2 and 182 degrees of freedom) performed on the indicated measure. Significance levels associated with these symbols are as follows: $\mathrm{ns}=p>.10 ;+=0.10<p<0.05 ;^{*}=p<.05 ;{ }^{* *}=p<.01{ }^{* * *}=p$ $<0.001 ; * * * *=p<0.0001$

pairs obtained in the narrative condition have been excluded for the sake of expository simplicity.)

Before turning to the Task 2 RT differences that are the main focus of this experiment, several things are worth noting about these data. First, participants in the neutral condition reproduced the typical pattern of cluster-status effects. In this condition, cluster retrieval was common $(67 \%)$ and the events in these clustered pairs often were causally related $(68 \%)$. Clustered events tended to be temporally proximate (median age difference $=3$ days) and nonclustered events tended to be temporally disparate (median age difference $=459$ days, Kruskal-Wallis Test $\left.\chi^{2}(1, N=613)=147.16, p<.0001\right)$. Clustered pairs also were more likely than nonclustered pairs to involve the same people ( $52 \%$ vs. $\left.29 \% ; \chi^{2}(1, N=613)=27.33, p<.0001\right)$ and to take place in the same location ( $55 \%$ vs. $34 \% ; \chi^{2}(1, N=613)=23.38, p<$ $.0001)$, whereas nonclustered pairs were more likely than clustered pairs to be related by some feature not listed in the relation menu $\left(33 \%\right.$ vs. $\left.14 \% ; \chi^{2}(1, N=613)=31.38, p<.0001\right)$. Finally, Task 2 RTs were marginally faster when the cueing event accessed a clus- 
ter-mate (median $\mathrm{RT}=7.46 \mathrm{~s})$ than when it did not (median $\mathrm{RT}=9.15$ s), one-tailed Kruskal-Wallis Test $\chi^{2}(1, N=612)=1.67, .10>p>.05$.

A second important point concerns the clustering data. As expected, clustering was more common in the same-narrative condition $(94 \%)$ than in the neutral condition $(67 \%)$ and more common in the neutral condition than in the non-narrative condition $(30 \%$, $F(2,182)=133.14, p<.0001, M S E=0.05$. This pattern of differences indicates that participants in the same-narrative and non-narrative groups generally were able to retrieve the appropriate type of event memory. However, these clustering rates also suggest that participants in the same-narrative condition found the task to be easier than did those in the non-narrative condition. Consistent with this claim, LSD tests indicate that cued events were retrieved more rapidly in the same-narrative condition than in the non-narrative condition (see Figure 2), and this was true regardless of whether or not the retrieved event was drawn from the same cluster as the cueing event: for clustered pairs, Kruskal-Wallis Test $\chi^{2}(1, N=604)=18.94$, $p<.0001$; and for nonclustered pairs, Kruskal-Wallis Test $\chi^{2}(1, N=$ 499) $=5.61, p<.05$.

The data presented in Figure 2 also indicate that (a) clustermates were retrieved faster in the same-narrative condition (median RT = $6.71 \mathrm{~s}$ ) than in the neutral condition (median RT $=7.46 \mathrm{~s}$ ), Kruskal-Wallis Test $\chi^{2}(1, N=1015)=6.48, p=.01$, and that $(b)$ nonclustered events were retrieved faster in the neutral condition (median RT $=9.15 \mathrm{~s}$ ) than in the non-narrative condition (median RT $=11.17 \mathrm{~s})$, Kruskal-Wallis Test $\chi^{2}(1, N=662)=11.14, p<.001$. For reasons developed above, the first of these RT differences indicates that cluster rates provide a conservative estimate of the underlying prevalence of event clusters in memory, and the second rules out biased search (or biased reconstruction) as the main reason for the frequent retrieval of clustermates.

\section{DISCUSSION}

In brief, the usual cluster-status effects were obtained in the neutral condition. Relative to this baseline, the retrieval restriction imposed by the Task 2 instructions had opposite effects on the performance in the same-narrative and non-narrative conditions. In the same-narrative condition, participants almost always were able to retrieve clustermates in response to event cues and they responded rapidly

when they did. In the non-narrative condition, event retrieval was 
slow, and the retrieved events sometimes conformed to the task requirements, but often did not. Despite explicit instructions to avoid "same-story" responses, 30\% of the cued events were drawn from the same cluster as the cueing event.

These results provide additional support for the notion that event clusters play a central role in organizing autobiographical memory at the event level. Indeed, the high cluster rates and short retrieval times observed in the same-narrative condition suggest that previously reported cluster rates underestimate the actual frequency of these structures. At the same time, RTs in the non-narrative condition suggest that clustermate access is not only common but also difficult to avoid. The fact that participants in this condition responded to event cues with clustermates on many trials also is consistent with this point.

There is, however, an alternative interpretation for the surprising level of same-cluster responses obtained in the non-narrative condition, one that could threaten the interpretation of cluster rates obtained under other conditions. Recall that events are considered to be members of the same cluster when participants indicate that the paired events are causally related, nested one within the other, or part of the same story. It could be that people are predisposed to perceive causal relations between events or that they sometimes make an effort to understand concurrently presented events as episodes drawn from the same story. If so, these tendencies could bias performance on the relation-coding task, making event clusters appear more common than they actually are. Cluster-status differences in RT, event dating, and (non-cluster defining) relation coding argue against this interpretation and indicate that clustered event pairs differ from nonclustered event pairs in many ways. In addition, there are data from a third experiment that demonstrate that people are not prone to perceive casual relations where they do not exist.

In this follow-up study, participants were presented with one set of 18 word cues during Task 1 and with a different set of 18 word cues during Task 2. In both tasks, participants were required to respond with the first specific personal event that came to mind. Task 3 was a modified version of the relation-coding task described above. During this task, participants were presented with event pairs created by randomly selecting (without replacement) one event description from Task 1 and one event description from Task 2 . As before, partic- 


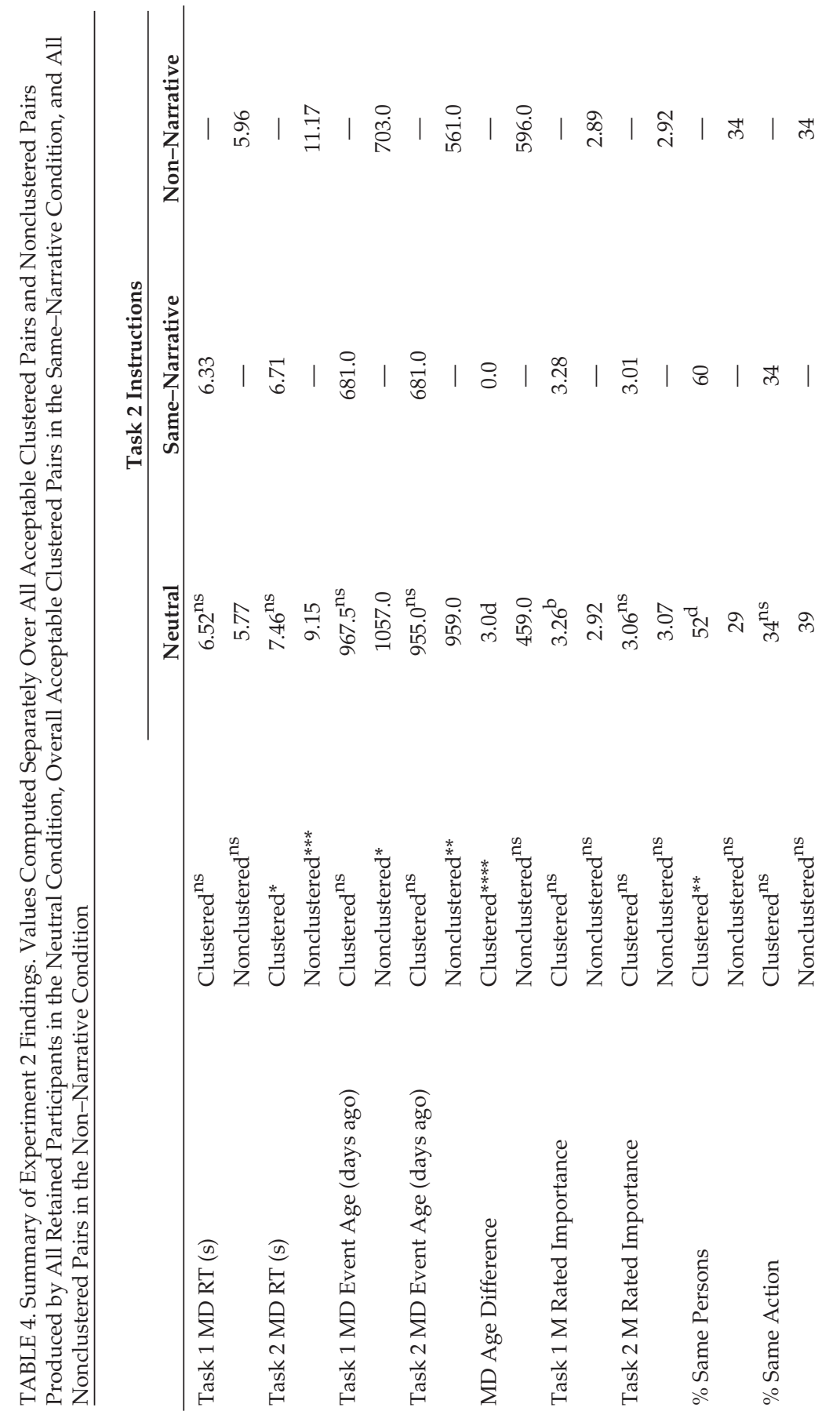




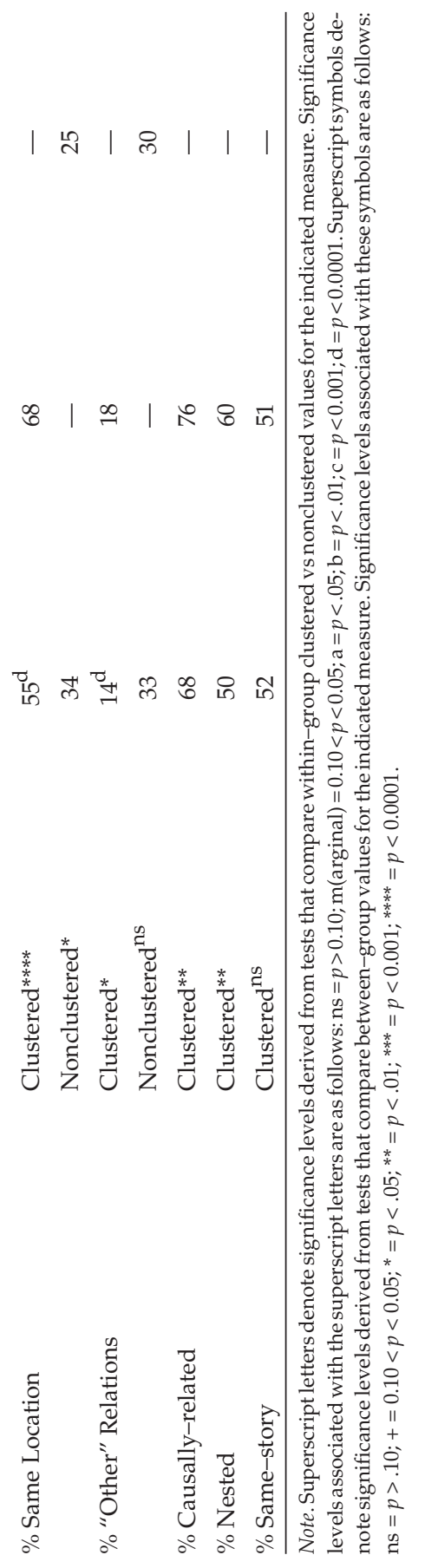


ipants were presented with a pair of events and were required to indicate how the events were related. In this case, participants had to indicate whether the events in the current pair referred to the same people, took place in the same location, or involved the same activity. They also were required to indicate whether one of the events caused the other and whether the events were related in some way not mentioned in the relation menu.

In this experiment, only $2 \%$ of 904 acceptable event pairs were judged to be causally related. Whereas $13 \%$ of these randomly matched event pairs involved the same people, $7 \%$ took place in the same location, $5 \%$ involved the same activity, and $17 \%$ overlapped in a way that was not specified by the relation menu. Given these results, it seems reasonable to conclude that the unexpectedly high cluster rate observed in the non-narrative condition is a reflection of the structural importance of the event cluster and not a consequence of a bias in the way that people perceive or report between-event relations.

\section{CONCLUSION}

In summary, two event-cueing experiments were described in this article. In Experiment 1, the age of the cueing event was manipulated between groups. Consistent with prior research, this experiment indicated that event memories are commonly embedded in story-like event clusters and that important event cues are somewhat more likely to elicit clustermates than unimportant event cues. It also turned out that the retrieval of clustermates was less common when the cueing events were from the distant past (i.e., childhood) than when they were from the recent past (i.e., high school) or the past week. In Experiment 2, retrieval restrictions were included as part of the event-cueing task as a way of determining whether a retrieval bias was behind the frequent recall of same-cluster event memories. Not only were between-group differences in RTs and cluster rates inconsistent with a biased-retrieval explanation, these data indicated that cluster rates produced under neutral instructions provide a conservative estimate of the underlying prevalence of event clusters in autobiographical memory.

These data raise some issues that should be addressed by future research. One of these concerns the relation between event age and cluster rates. As noted in the discussion following Experiment 1 , it should be possible to use a cross-sectional design to select between a developmental-encoding account of this phenomenon 
and a simple account based on forgetting. A second issue concerns the processes that create and maintain event clusters. Earlier in this article, the prevalence of event clusters and the relationship between clustering and event importance has been taken as evidence for a matter-of-course account of clustering. In other words, it appears that event clusters are created as a by-product of the processes required to coordinate goal-directed behavior over time. At this point, what is needed is more information about the exact nature of these processes and their mnemonic impact. In this context, it is encouraging that clustering is common even when events are very recent. This raises the possibility that processes that create and maintain event clusters might be studied in the laboratory or at least very near to it.

\section{REFERENCES}

Anderson, S. J., \& Conway, M. A. (1993). Investigating the structure of specific autobiographical memories. Journal of Experimental Psychology: Learning, Memory, \& Cognition, 19, 1-19.

Barclay, C. R. (1996). Autobiographical remembering: Narrative constraints on objectified selves. In D. C. Rubin (Ed.), Remembering our past (pp. 94-125). Cambridge: Cambridge University Press.

Barsalou, L. W. (1988). The content and organization of autobiographical memos. In U. Neisser \& E. Winograd (Eds.) Remember reconsidered: ecological and traditional approaches to memory (pp. 193-243). New York: Cambridge University Press.

Brown, N. R. (1990). Organization of public events in long-term memory. Journal of Experimental Psychology: General, 119, 297-314.

Brown, N. R. (1993). Response times, retrieval strategies, and the investigation of autobiographical memory. In T. K. Srull \& R. S. Wyer (Eds.), Mental representation of trait and autobiographical knowledge of the self: Advances in social cognition, Vol. 5 (pp. 61-68). Hillsdale, NJ: Erlbaum.

Brown, N. R., \& Schopflocher, D. (1998a). Event cueing, event clusters, and the temporal distribution of autobiographical memories. Applied Cognitive Psychology, 12, 305-319.

Brown, N. R., \& Schopflocher, D. (1998b). Event clusters: An organization of personal events in long-term memory. Psychological Science, 9, 470-475.

Bruner, J. (1991). The narrative construction of reality. Critical Inquiry, Autumn, 1-21.

Bruner, J., \& Feldman, C. F. (1996). Group narrative as a cultural context of autobiography. In D. C. Rubin (Ed.), Remembering our past (pp. 291-317). Cambridge: Cambridge University Press.

Burt, C. D. R., Mitchell, D. A., Raggatt, P. T. F., Jones, C. A., \& Cowan, T. M. (1995). A 
snapshot of autobiographical memory retrieval characteristics. Applied Cognitive Psychology, 9, 61-74.

Conway, M. A. (1996). Autobiographical knowledge and autobiographical memories. In D. C. Rubin (Ed.), Remembering Our Past (pp. 67-93). Cambridge: Cambridge University Press.

Fitzgerald, J. M. (1980). Sampling autobiographical memory reports in adolescents. Developmental Psychology, 16, 675-676.

Hayes-Roth, B., \& Hayes-Roth, F. (1979). A cognitive model of planning. Cognitive Science, 3, 275-310.

Kolodner, J. L. (1983). Maintaining organization in a dynamic long-term memory. Cognitive Science, 7, 243-280.

Linton, M. (1986). Ways of searching the contents of memory. In D. C. Rubin (Ed.), Autobiographical memory (pp. 50-67). New York: Cambridge University Press.

Nelson, K. (1993). The psychological and social origins of autobiographical memory. Psychological Science, 3, 7-14.

Norman, D. A. \& Bobrow, D. C. (1979). Descriptions: An intermediate stage in memory retrieval. Cognitive Psychology, 11, 107-123.

Reiser, B. J. (1983). Contexts and indices in autobiographical memory. (Technical Report 24). New Haven CT: Yale University, Department of Cognitive Science.

Reiser, B. J., Black, J. B., \& Abelson, R. P. (1985). Knowledge structures in the organization and retrieval of autobiographical memories. Cognitive Psychology, 17, 89-137.

Robinson, J. A. (1976). Sampling autobiographical memory, Cognitive Psychology, 8, 578-595.

Robinson, J. A. (1992). First experience memories: Context and functions in personal histories. In M. A. Conway, D. C. Rubin, H. Spinnler, \& W. A. Wagenaar (Eds.), Theoretical Perspective on Autobiographical Memory (pp. 223-239). Dordrecht, The Netherlands: Kluwer.

Robinson, J. A, \& Taylor, L. R. (1998). Autobiographical memory and self-narratives: A tale of two stories. In C. P. Thompson \& D. J. Herrmann (Eds.), Autobiographical memory: Theoretical and applied perspectives. (pp. 125-143). Mahwah, NJ: Erlbaum.

Roese, N. J., \& Olson, J. M. (1995). Counterfactual thinking: A critical overview. In N. J. Roese \& J. M. Olson (Eds.), What might have been: The social psychology of counterfactual thinking (pp. 1-55). Hillsdale: Erlbaum.

Rubin, D. C. (1982). On the retention function for autobiographical memory. Journal of Verbal Learning and Verbal Behavior, 21, 21-38.

Sato, K. (2002). Changes in the temporal organization of autobiographical memories. Psychological Reports, 91, 1074-1078.

Schank, R. C. (1982). Dynamic Memory. Hillsdale: Erlbaum.

Schank, R. C., \& Abelson, R. P. (1977). Scripts, plans, goals, and understanding. Hillsdale: Erlbaum.

Schank, R. C., \& Abelson, R. P. (1995). Knowledge and memory: The real story. In R. S. Wyer (Ed.), Advances in social cognition, Vol. VII (pp. 1-85). Hillsdale: Erlbaum.

Thompson, C. P., Skowronski, J. J., \& Betz, A. L. (1993). The use of partial information in dating personal events. Memory $\mathcal{E}$ Cognition, 21, 352-360.

Trabasso, T., \& van den Broek, P. (1985). Causal thinking and the representation of narrative events. Journal of Memory and Language, 24, 612-630 
Williams, M. D., \& Hollan, J. D. (1981). The process of retrieval from very long-term memory. Cognitive Science, 5, 87-119.

Wright, D. B., \& Nunn, J. A. (2000). Similarities within event clusters in autobiographical memory. Applied Cognitive Psychology, 14, 479-489. 